\title{
Factors Associated With Non-Adherence To Antidiabetic Medication Among Patients at Mbarara Regional Referral Hospital, Mbarara, Uganda
}

\author{
Karekoona Faisal \\ Mbarara University of Science and Technology \\ TADELE MEKURIYA ( $\nabla$ mtadele@must.ac.ug ) \\ Mbarara University of Science and Technology \\ Jonans Tusiimire \\ Mbarara University of Science and Technology
}

\section{Research Article}

Keywords: Non-adherence, Contributing factors, Prevalence, antidiabetic drugs, Uganda

Posted Date: June 17th, 2021

DOI: https://doi.org/10.21203/rs.3.rs-571953/v1

License: (c) This work is licensed under a Creative Commons Attribution 4.0 International License.

Read Full License 


\section{Abstract \\ Background}

Non-adherence is a major concern in treatment of diabetes mellitus and undermines the goals of treatment. The objective of this study was to determine the magnitude of non-adherence and its contributing factors among diabetes mellitus patients attending the Diabetes mellitus clinic at Mbarara Regional Referral Hospital.

\section{Objective}

To assess the factors contributing to non-adherence to antidiabetic medication among diabetes mellitus patients in the Diabetic clinic at Mbarara Regional Referral Hospital

\section{Methods}

A descriptive cross-sectional study was done at the Diabetes clinic, Mbarara Regional Referral Hospital, between July and October 2020. Study participants were systemically sampled and data regarding their medication non-adherence was collected using a structured questionnaire, based on the Hill-Bone medication adherence scale. Data entry was done using Microsoft Excel Version 2010 and analysis was carried out using STATA version 13.

\section{Results}

A total of 257 participants were recruited with $100 \%$ response rate. More than one third $(98,38.1 \%)$ of the participants were non-adherent to their antidiabetic medication. Age above 60 years $(A O R=6.26,95 \% \mathrm{Cl}=$ $1.009-39.241, P=0.049)$, and duration of diabetes mellitus above 5years $(A O R=1.87,95 \% \mathrm{Cl}=1.034-$ $3.392, \mathrm{P}=0.038$ ) were independently associated with non-adherence to antidiabetic medication.

\section{Conclusion}

The prevalence of non-adherence to antidiabetic medication was higher than that revealed in previous studies in Uganda. Patients with age above 60 years were six times more likely to be non-adherent to their antidiabetic medications. Patient education is important to address the challenge of medication nonadherence.

\section{Introduction}


Diabetes mellitus (DM) is a major public health issue globally. Current estimates indicate that nearly 415 million people are affected and is set to escalate to 642 million by the year 2040, a further 193 million people with diabetes remain undiagnosed due to the often mild or asymptomatic nature of this condition especially in type 2 DM (T2DM) (1)

Adherence to anti-diabetic medication is one of the major pillars of health service excellence and is defined as the proportion of the prescribed doses of the drug actually taken by a patient over a specified period of time or the extent to which the patient is taking their medicines as prescribed by a health care professional (2).

Non-adherence to antidiabetic medication remains the most common reason for poor health outcomes among people with diabetes. The levels of non-adherence to antidiabetic recommendations are highly variable, but have significant effects on diabetes outcomes and the effectiveness of treatments $(3,4)$. Continuous evaluation of adherence is vital to identify factors and barriers contributing to non-adherence and its better management through timely identification of contributing factors and provision of individualized suitable recommendations that are essential for better healthcare management (5).

Poor persistence with and adherence to antidiabetic medication exposes the patient to disease complications with fatal consequences, including failure to achieve glycemic control goals (6), Contributes to the suboptimal glycemic control and continues to be one of the major barriers to effective diabetes mellitus management (7).

A study in India, established that $55.14 \%$ of the study participants were non-adherent and the Major contributing factors of non-adherence to antidiabetic treatment were ignorance for life style modification i.e. $83.78 \%$. Among them, $59.48 \%$ didn't take the prescribed medicine in time, most of them $85.71 \%$ didn't follow diabetes diet and less than half (46.61\%) didn't monitor blood glucose level regularly due to poor self-discipline. Gender, occupation and educational status were the significant contributors to nonadherence (8).

In Saudi Arabia, a therapeutic non-compliance prevalence of $67.9 \%$ (i.e. $69.34 \%$ in males and $65.45 \%$ in females) was largely associated with females, illiteracy, urban population, irregularity of follow-ups, nonadherence to antidiabetic medication, non-adherence to exercise regimen, insulin, and insulin with oral Metformin (9).

A study done by Gertrude Afriye and others at the University of Ghana among 259 patients within the ages of 26 years and 88 years established a proportion of non-adherence to antidiabetic medication of $34.7 \%$. and associated factors that turned out to be statistically significant for non-adherence included; Age, educational level, presence of comorbidities, and financial support (10). while in Ethiopia at Adama Hospital medical college, The prevalence of non-adherence was $58.6 \%(95 \% \mathrm{Cl}: 54.7,62.4)$ and major depressive disorder, one or more diabetes mellitus complications, and average income greater than 1000 birr were found to be independent predictors of medication non-adherence respectively (11). 
At a general hospital in Ethiopia, a non-adherence to antidiabetic medication prevalence of $31.2 \%$ amongst diabetes mellitus patients was established while side effects of medications, complexity of regimen, failure to remember, educational level and monthly income were major associated factors identified in the study (12).

Fewer studies on non-adherence to antidiabetic medication have been conducted in Uganda. However, a non-adherence prevalence to antidiabetic medication of $28.9 \%$ at Mulago hospital in Kampala district was mainly associated with female gender, illiteracy, low social economic status, poor handwriting on prescriptions, and delayed intervals to follow up on treatments (13). Hence, this study aimed at determining the prevalence and identifying factors associated with non-adherence to anti-diabetes medication among patients in the Diabetes mellitus clinic at Mbarara regional Referral hospital, south western Uganda.

\section{Methods And Materials}

\section{Study setting and population}

The study was conducted in the Diabetes mellitus clinic at Mbarara Regional Referral Hospital (MRRH) located in Mbarara City, Mbarara district, south western region, Uganda from July to October 2020; it is a government owned referral and teaching hospital for Mbarara University Medical School with patient capacity of approximately of 350 beds. The general medical care in the hospital is free and the Diabetes mellitus clinic is one of the major ambulatory clinics operated once a week within the hospital. The study population was diabetes mellitus patients (both type 1 and type 2) who attended the Diabetes clinic at MRRH between July and October, 2020.

\section{Study design}

Descriptive cross-sectional study was done, Outcome variables were Non-adherence prevalence and Factors contributing to non-adherence to antidiabetic medication

\section{Sampling procedure and sample size Estimation}

The sample size was calculated using finite population formula and the prevalence of non-adherence to antidiabetic medication of $28.9 \%$ in Uganda by (13) was used for this calculation. Using formula (14);

$$
n=\frac{\left(z_{\alpha}\right)^{2} x p x(1-p)}{e^{2}}
$$

Where:

$Z a=$ Standard deviation at $95 \%$ confidence interval corresponding to 1.96 
$p=$ assumed population of diabetes mellitus patients who are non-adherent to treatment, results of a study at Mulago hospital (13) were used, so $P=28.9 \%$

$1-\mathrm{P}=$ is the probability of diabetes mellitus patients who were adhering to their antidiabetic medication, therefore $1-\mathrm{P}=71.1 \%$

$\mathrm{e}=$ level of precision (in proportion of one, therefore considering $5 \%, e=0.05$ )

$\mathrm{n}=$ sample size estimation of patients with diabetes mellitus

Estimated sample size, $N=\frac{(1.96)^{2} \times 0.289 \times(1-0.289)}{0.05^{2}}=316$

Estimated size $(\mathrm{N})=316$

Number of active patients at the Diabetes clinic (MRRH) was approximately 1,074 (source; Diabetes clinic records). The finite population correction factor ( $n$ )

$\mathrm{n}=\frac{n o N}{n o+(N-1)}$

Total number of active patients (no) $=1074$ (adopted from Diabetes clinic records)

Estimated sample size $(\mathrm{N})=316$

Therefore, sample size $(n)=245$ patients

Adding $5 \%$ for incomplete information and withdraw from the study, $245 \times 0.05=12$

Total target sample $=245+12=257$.

Systematic random sampling technique was used to select study participants.

Sampling interval $=1074$ / $257=4$, interviewed every fourth patient on the clinic appointment list starting from a number randomly selected from 1 to 4

\section{Data collection procedure}

Structured investigator-administered pre-tested questionnaire was used for each participant to collect information on socio-demographic and adherence characteristics. A check list was used to document diseases (Diabetes mellitus and other comorbidities) and drugs' (antidiabetic medication) information for each patient.

A detailed history was acquired (English), translated where necessary for patients who do not understand English. 
Non-adherence to antidiabetic medication was assessed based on the Hill-Bone Medication Adherence scale (15).

\section{Data Quality control}

A registered nurse in the Diabetes clinic was recruited and well trained on data collection techniques. The data collection instruments were pretested on five (5) patients in the Diabetes clinic to identify possible sources of errors that would arise during data collection. Those five patients were not part of the sample population and the questionnaire was administered to them to measure the inter-respondent agreement. The agreement of more than $78 \%$ was a measure that the items of the questionnaire would provide a picture on factors associated with non - adherence to antidiabetic medication in the study population.

Inclusion and exclusion criteria were strictly adhered to. A common pretested questionnaire which was edited prior to its use was used for data collection. The questionnaire was also checked for completeness before data collection to ensure valid data was obtained. Identification of participants was done by means of numerical codes. Details of participants were kept under lock and key for privacy and confidentiality purpose throughout the course of the study. There was no disclosure of participants' identity to the public and all identities were removed before publication.

\section{Data management and statistical analysis}

Data on questionnaires was checked for completeness, coded and entered in Microsoft Excel version 2010 , and then exported to stata version 13. Socio-demographic and clinical factors were summarized as means and standard deviation (for continuous variables). Percentages and frequencies were used for categorical variables. Descriptive statistics (means, frequencies and percentages) were used to determine prevalence of non-adherence and patient and disease information. The factors associated with nonadherence to antidiabetic medication were identified using logistic regression. Binary logistic Regression was carried out using stata. Prevalence Ratios were used to compare the prevalence of non-adherence across the independent variables. Factors with $p$-value $\leq 0.20$ at bivariate logistic regression and with biological plausibility were considered for multivariate analysis. Those factors with $p$-value $<0.05$ were considered significant.

\section{Ethical considerations}

This study was conducted in accordance with the Declaration of Helsinki (16). Approval to carry out the study was acquired from the Department of Pharmacy, Faculty of Medicine and finally from Mbarara University Research Ethics Committee (MUST-REC). After approval by the MUST-REC (Letter with reference number MUREC 1/7), permission was thought from the administration of Mbarara Regional Referral Hospital prior to data collection in the Diabetes mellitus clinic.

\section{Precautions for prevention of Covid 19 transmission}


Participants who were initially screened for body temperature and possible signs and symptoms of covid 19 were considered for data collection. These were further given more information on how covid 19 is transmitted and finally preventive measures of hand washing, wearing face mask and social distancing were always emphasized before any interaction with the study participants.

\section{Results}

The study had a response rate of $100 \%$ of 257 respondents.

\section{Socio-Demographic Characteristics of Participants.}

The study involved 257 participants consisting of 168 (65.4\%) females and 89(34.6) males. Majority $(64.6 \%)$ of the participants were in the age range of $25-60$ years $(53 \pm 13.3)$; almost one quarter $(23.4 \%)$ had no formal education and almost one half $(122,47.5 \%)$ were protestants and one third $(85,33.1 \%)$ were farmers. Majority $(192,74.7 \%)$ had no family history of diabetes mellitus (Table 1 ). 
Table 1

Socio demographic characteristics of participants in the Diabetes clinic at Mbarara Regional Referral Hospital from July and October 2020.

\begin{tabular}{|c|c|c|}
\hline Variable & Categories & Frequency $\mathrm{N}(\%)$ \\
\hline \multirow[t]{3}{*}{ Age (years) } & $18-24$ & $9(3.5)$ \\
\hline & $25-60$ & $166(64.6)$ \\
\hline & Above 60 & $82(31.9)$ \\
\hline \multirow[t]{2}{*}{ Gender } & Female & $168(65.4)$ \\
\hline & Male & $89(34.6)$ \\
\hline \multirow[t]{4}{*}{ Education } & Not educated & $60(23.4)$ \\
\hline & Primary/Intermediate & $106(41.3)$ \\
\hline & Secondary & $56(21.8)$ \\
\hline & Tertiary & $35(13.6)$ \\
\hline \multirow[t]{4}{*}{ Religion } & Protestant/Anglican & $122(47.5)$ \\
\hline & Catholic & $80(31.1)$ \\
\hline & Muslims & $39(15.2)$ \\
\hline & Pentecostal & $16(6.2)$ \\
\hline \multirow[t]{4}{*}{ Tribe } & Banyankole & 187(72.8) \\
\hline & Bakiga & $30(11.7)$ \\
\hline & Baganda & $27(10.5)$ \\
\hline & Others & $13(5.1)$ \\
\hline \multirow[t]{2}{*}{ Family History of Diabetes } & Yes & $65(25.3)$ \\
\hline & No & 192(74.7) \\
\hline \multirow[t]{4}{*}{ Employment Status } & Peasant/farmer & $85(33.1)$ \\
\hline & Unemployed & $63(24.5)$ \\
\hline & Employed & $58(22.6)$ \\
\hline & Entrepreneur & $51(19.8)$ \\
\hline
\end{tabular}

\section{Life Style and Disease Characteristics}

Majority $(253,98.4 \%)$ of the study participants had Type 2 diabetes mellitus and more than one half ( 160 , $62.3 \%)$ had been diagnosed with diabetes mellitus within five years. More than three quarters (214, $83.3 \%)$ of the participants had comorbidities of which hypertension $(143,66.8 \%)$ was the most common. 
Majority $(148,81.3 \%)$ of the participants had diabetes mellitus related complications of which 119 $(79.9 \%)$ had been diagnosed with neuropathy. More than one half $(142,55.3 \%)$ of the participants were on Metformin and glibenclamide antidiabetic regimen (Table 2). 
Table 2

life style and disease characteristics among patients in the diabetes mellitus clinic at Mbarara regional referral hospital from July to October 2020.

\begin{tabular}{|c|c|c|c|}
\hline Variable & Category & Frequency & Percentage (\%) \\
\hline \multirow[t]{2}{*}{ Alcohol intake } & Yes & 11 & 4.3 \\
\hline & No & 246 & 95.7 \\
\hline \multirow[t]{2}{*}{ Physical exercise } & Yes & 168 & 65.37 \\
\hline & No & 89 & 34.6 \\
\hline \multirow[t]{2}{*}{ Smoking tobacco } & Yes & 5 & 1.9 \\
\hline & No & 252 & 98.1 \\
\hline \multirow[t]{3}{*}{ BMI } & Normal & 99 & 38.5 \\
\hline & Overweight & 93 & 36.2 \\
\hline & Obese & 65 & 25.3 \\
\hline \multirow[t]{2}{*}{ Type of Diabetes } & Type 1 & 4 & 1.6 \\
\hline & Type 2 & 253 & 98.4 \\
\hline \multirow[t]{2}{*}{ Duration Diabetes } & 5 Years \& Below & 160 & 62.3 \\
\hline & Above 5 Years & 97 & 37.7 \\
\hline \multirow[t]{2}{*}{ Presence of Comorbidity } & Yes & 214 & 83.3 \\
\hline & No & 43 & 16.7 \\
\hline \multirow[t]{3}{*}{ Type of Comorbidity } & Hypertension & 143 & 66.8 \\
\hline & HIV & 52 & 24.3 \\
\hline & Others & 19 & 18.4 \\
\hline \multirow[t]{2}{*}{ Presence of Diabetes complication(s) } & Yes & 148 & 81.3 \\
\hline & No & 109 & 18.7 \\
\hline \multirow[t]{3}{*}{ Type of Diabetes Complication } & Neuropathy & 119 & 79.9 \\
\hline & Retinopathy & 18 & 12.1 \\
\hline & Others & 11 & 7.4 \\
\hline \multirow[t]{3}{*}{ Antidiabetics used } & Metformin & 35 & 13.6 \\
\hline & Insulin & 36 & 14.0 \\
\hline & Glibenclamide & 1 & 0.4 \\
\hline
\end{tabular}




\begin{tabular}{|llll|}
\hline Variable & Category & Frequency & Percentage (\%) \\
\hline & Metformin \& Insulin & 43 & 16.7 \\
\cline { 2 - 4 } & Metformin \& Glibenclamide & 142 & 55.1 \\
\hline
\end{tabular}

\section{Prevalence of non-adherence}

Over one third $(98,38.1 \%)$ of the study participants were non adherent to antidiabetic medication (Fig. 1).

\section{Reasons for Non-adherence to Antidiabetic Medication}

More than one quarter $(50,25.5 \%)$ of the study participants reported that drug(s) being expensive was the cause for antidiabetic medication non-adherence and Less than one quarter $(38,19.4 \%)$ ascribed nonadherence to not understanding instruction(s) on how to take prescribed medicine(s) and less than one quarter $(32,16.3 \%)$ of the study participants attributed non-adherence to unavailability of the drug product to them (Table 4).

Table 3

Patients' reasons for non-adherence among participants in the Diabetes clinic at Mbarara Regional Referral Hospital from July to October 2020.

\begin{tabular}{|lll|}
\hline Factors & Frequency & Percentage (\%) \\
\hline Drug is expensive to buy on daily basis & 50 & 25.5 \\
\hline Doesn't understand prescriptions & 38 & 19.4 \\
\hline Drug product not available & 32 & 16.3 \\
\hline Forgets to take the drug & 24 & 12.2 \\
\hline Patient prefers not to take the drug & 24 & 12.2 \\
\hline Not willing to take the drug & 16 & 8.2 \\
\hline Cannot swallow or administer drug & 10 & 5.2 \\
\hline Others & 02 & 1.0 \\
\hline
\end{tabular}

\section{Factors associated with non-adherence to antidiabetic medication}

\subsubsection{Bivariate analysis}

Bivariate logistic regression analysis revealed that: - age range above 60 years $(\mathrm{COR}=5.47,95 \% \mathrm{Cl}=$ 1.068-27.993, $\mathrm{P}=0.041$ ) and living with diabetes mellitus for duration above five years since diagnosis $(\mathrm{COR}=2.15,95 \% \mathrm{Cl}=1.281-3.624, \mathrm{P}=0.004)$; were significantly associated with antidiabetic medication non adherence. 
Table 4

Results of bivariate analysis for the association between contributing factors and diabetic medication non-adherence among participants in the Diabetic clinic at Mbarara Regional Referral Hospital between July to October 2020.

\begin{tabular}{|c|c|c|c|c|c|c|}
\hline \multirow{3}{*}{ Variable } & \multirow{3}{*}{ Category } & \multicolumn{2}{|c|}{ Non-Adherence } & \multicolumn{3}{|c|}{ Bivariate analysis } \\
\hline & & No & Yes & COR & $95 \% \mathrm{Cl}$ & P- \\
\hline & & $\begin{array}{l}N=159 \\
(\%)\end{array}$ & $\begin{array}{l}N= \\
98(\%)\end{array}$ & & & \\
\hline \multirow[t]{3}{*}{ Age (years) } & $18-24$ & $7(77.8)$ & $2(22.2)$ & 1 & & \\
\hline & $25-60$ & $\begin{array}{l}120 \\
(72.3)\end{array}$ & $\begin{array}{l}46 \\
(27.7)\end{array}$ & 1.34 & $\begin{array}{l}0.269- \\
6.697\end{array}$ & 0.720 \\
\hline & Above 60 & $32(39.0)$ & $\begin{array}{l}50 \\
(61.0)\end{array}$ & 5.47 & $\begin{array}{l}1.068- \\
27.993\end{array}$ & $0.041^{*}$ \\
\hline \multirow[t]{3}{*}{ BMI } & Normal & $60(60.6)$ & $\begin{array}{l}39 \\
(39.4)\end{array}$ & 1 & & \\
\hline & Overweight & $55(59.1)$ & $\begin{array}{l}38 \\
(40.9)\end{array}$ & 1.06 & $\begin{array}{l}0.597- \\
1.894\end{array}$ & 0.84 \\
\hline & Obese & $44(67.7)$ & $\begin{array}{l}21 \\
(32.3)\end{array}$ & 0.73 & $\begin{array}{l}0.380- \\
1.418\end{array}$ & 0.36 \\
\hline \multirow[t]{2}{*}{ Gender } & Male & $48(53.9)$ & $\begin{array}{l}41 \\
(46.1)\end{array}$ & & & \\
\hline & Female & 111(66.1) & $\begin{array}{l}57 \\
(33.9)\end{array}$ & 0.60 & $\begin{array}{l}0.356- \\
1.016\end{array}$ & $0.058 *$ \\
\hline \multirow[t]{4}{*}{ Education } & Primary/Intermediate & $71(67.0)$ & $\begin{array}{l}35 \\
(33.0)\end{array}$ & 1 & & \\
\hline & Not educated & $32(53.3)$ & $\begin{array}{l}28 \\
(46.7)\end{array}$ & 1.78 & $\begin{array}{l}0.928- \\
3.396\end{array}$ & 0.083 \\
\hline & Secondary & $36(64.3)$ & $\begin{array}{l}20 \\
(35.7)\end{array}$ & 1.13 & $\begin{array}{l}0.571- \\
2.225\end{array}$ & 0.730 \\
\hline & Tertiary & $20(57.1)$ & $\begin{array}{l}15 \\
(42.9)\end{array}$ & 1.52 & $\begin{array}{l}0.695^{-} \\
3.327\end{array}$ & 0.293 \\
\hline \multirow[t]{2}{*}{$\begin{array}{l}\text { Physical exercise } \\
\text { performance }\end{array}$} & Yes & $\begin{array}{l}104 \\
(61.9)\end{array}$ & $\begin{array}{l}64 \\
(38.1)\end{array}$ & 1 & & \\
\hline & No & $55(61.8)$ & $\begin{array}{l}34 \\
(38.2)\end{array}$ & 1.00 & $\begin{array}{l}0.592- \\
1.705\end{array}$ & 0987 \\
\hline Smoking tobacco & Yes & $4(80.0)$ & $1(20.0)$ & 1.00 & & \\
\hline
\end{tabular}

NB: those with a * had a $p$-value $\leq \mathbf{0 . 2 0}$ and were considered for multivariate logistic regression analysis 


\begin{tabular}{|c|c|c|c|c|c|c|}
\hline & \multirow[b]{2}{*}{ No } & \multicolumn{2}{|c|}{ Non-Adherence } & \multicolumn{3}{|c|}{ Bivariate analysis } \\
\hline & & $\begin{array}{l}155 \\
(61.5)\end{array}$ & $\begin{array}{l}97 \\
(38.3)\end{array}$ & 2.50 & $\begin{array}{l}0.276- \\
22.726\end{array}$ & 0.415 \\
\hline \multirow[t]{2}{*}{ Alcohol consumption } & Yes & $4(36.4)$ & $7(63.6)$ & 1 & & \\
\hline & No & $\begin{array}{l}155 \\
(63.0)\end{array}$ & $\begin{array}{l}91 \\
(37.0)\end{array}$ & 0.34 & $\begin{array}{l}0.096- \\
1.177\end{array}$ & $0.088 *$ \\
\hline \multirow[t]{2}{*}{ Type of Diabetes } & Type 1 & $2(50.0)$ & $2(50.0)$ & 1 & & \\
\hline & Type 2 & $\begin{array}{l}157 \\
(62.1)\end{array}$ & $\begin{array}{l}96 \\
(37.9)\end{array}$ & 0.61 & $\begin{array}{l}0.148- \\
2.482\end{array}$ & 0.487 \\
\hline \multirow[t]{2}{*}{$\begin{array}{l}\text { Duration of diabetes } \\
\text { (years) }\end{array}$} & 5 years \& below & $\begin{array}{l}110 \\
(68.7)\end{array}$ & $\begin{array}{l}50 \\
(31.3)\end{array}$ & 1 & & \\
\hline & Above 5 years & $49(50.5)$ & $\begin{array}{l}48 \\
(49.5)\end{array}$ & 2.15 & $\begin{array}{l}1.281- \\
3.624\end{array}$ & $0.004^{*}$ \\
\hline \multirow[t]{2}{*}{$\begin{array}{l}\text { Presence of } \\
\text { Complications }\end{array}$} & Yes & $\begin{array}{l}132 \\
(63.3)\end{array}$ & $77(36.7)$ & 1 & & \\
\hline & No & $27(56.3)$ & $\begin{array}{l}21 \\
(43.7)\end{array}$ & 1.33 & $\begin{array}{l}0.706- \\
2.518\end{array}$ & 0.375 \\
\hline \multirow{2}{*}{$\begin{array}{l}\text { Presence of } \\
\text { Comorbidities }\end{array}$} & Yes & $85(62.5)$ & $\begin{array}{l}51 \\
(37.5)\end{array}$ & 1 & & \\
\hline & No & $74(61.2)$ & $\begin{array}{l}47 \\
(38.8)\end{array}$ & 1.05 & $\begin{array}{l}0.424- \\
0.849\end{array}$ & 0.825 \\
\hline \multirow[t]{5}{*}{ Religion } & Protestants & $76(62.3)$ & $\begin{array}{l}46 \\
(37.7)\end{array}$ & 1 & & \\
\hline & Catholics & $53(66.2)$ & $\begin{array}{l}27 \\
(33.8)\end{array}$ & 0.84 & $\begin{array}{l}0.466- \\
1.519\end{array}$ & 0.567 \\
\hline & Muslims & $19(48.7)$ & $\begin{array}{l}20 \\
(51.3)\end{array}$ & 1.74 & $\begin{array}{l}0.841- \\
3.597\end{array}$ & $0.136^{*}$ \\
\hline & Pentecostal & $8(66.7)$ & $4(33.3)$ & 0.83 & $\begin{array}{l}0.236- \\
2.897\end{array}$ & 0.765 \\
\hline & Others | & $3(75.0)$ & $1(25.0)$ & 0.55 & $\begin{array}{l}0.056- \\
5.452\end{array}$ & 0.610 \\
\hline \multirow[t]{2}{*}{ Employment } & Employed & $33(56.9)$ & $\begin{array}{l}25 \\
(43.1)\end{array}$ & 1 & & \\
\hline & Peasant/farmer & $45(52.9)$ & $\begin{array}{l}40 \\
(47.1)\end{array}$ & 1.17 & $\begin{array}{l}0.599- \\
2.297\end{array}$ & 0.641 \\
\hline
\end{tabular}

NB: those with a * had a $p$-value $\leq \mathbf{0 . 2 0}$ and were considered for multivariate logistic regression analysis 


\begin{tabular}{|c|c|c|c|c|c|c|}
\hline & \multirow[b]{2}{*}{ Entrepreneur } & \multicolumn{2}{|c|}{ Non-Adherence } & \multicolumn{3}{|c|}{ Bivariate analysis } \\
\hline & & $30(71.4)$ & $\begin{array}{l}12 \\
(28.6)\end{array}$ & 0.53 & $\begin{array}{l}0.226- \\
1.232\end{array}$ & $0.140 *$ \\
\hline & Un employed & $45(71.4)$ & $\begin{array}{l}18 \\
(28.6)\end{array}$ & 0.53 & $\begin{array}{l}0.248- \\
1.123\end{array}$ & $0.097^{*}$ \\
\hline & Others & $6(66.7)$ & $3(33.3)$ & 0.66 & $\begin{array}{l}0.150- \\
2.899\end{array}$ & 0.582 \\
\hline \multirow[t]{4}{*}{ Tribe } & Banyankole & $117(62.6)$ & $\begin{array}{l}70 \\
(37.4)\end{array}$ & 1 & & \\
\hline & Bakiga & $18(60.0)$ & $12(40.0)$ & 1.11 & $\begin{array}{l}0.506- \\
2.451\end{array}$ & 0.788 \\
\hline & Buganda & 14(51.9) & $13(48.1)$ & 1.55 & $\begin{array}{l}0.689- \\
3.492\end{array}$ & 0.288 \\
\hline & Others & 10(76.9) & $3(23.1)$ & 0.50 & $\begin{array}{l}0.133- \\
1.884\end{array}$ & 0.307 \\
\hline \multirow[t]{4}{*}{$\begin{array}{l}\text { Antidiabetic regimen } \\
\text { used }\end{array}$} & Metformin & $24(66.7)$ & $\begin{array}{l}12 \\
(33.3)\end{array}$ & 1 & & \\
\hline & Insulin & 19 (52.8) & $\begin{array}{l}17 \\
(47.2)\end{array}$ & 1.95 & $\begin{array}{l}0.741- \\
5.140\end{array}$ & $0.176^{\star}$ \\
\hline & Metformin \& insulin & $31(72.1)$ & $\begin{array}{l}12 \\
(27.9)\end{array}$ & 0.84 & $\begin{array}{l}0.318- \\
2.242\end{array}$ & 0.735 \\
\hline & $\begin{array}{l}\text { Metformin \& } \\
\text { Glibenclamide }\end{array}$ & $85(59.9)$ & $\begin{array}{l}57 \\
(40.1)\end{array}$ & 1.46 & $\begin{array}{l}0.664- \\
3.219\end{array}$ & 0.344 \\
\hline
\end{tabular}

\section{Multivariate analysis}

Multivariate logistic regression involving variables that had $p$-value $\leq 0.20$ at bivariate logistic regression was done. Age range above 60 years $(\mathrm{AOR}=6.26,95 \% \mathrm{Cl}=1.009-39.241, \mathrm{P}=0.049)$ and Duration of diabetes mellitus above 5 years since diagnosis $(A O R=1.87,95 \% \mathrm{Cl}=1.034-3.391, \mathrm{P}=0.038)$ were associated with antidiabetic medication non adherence and had a statistically significant relationship. Not being employed $(\mathrm{AOR}=0.37,95 \% \mathrm{Cl}=0.139-0.977, \mathrm{P}=0.045)$ was protective and therefore, less likely to lead to antidiabetic medication non-adherence. 
Table 5

Multivariate analysis for the association between contributing factors and diabetic medication nonadherence among participants in the Diabetic clinic at Mbarara Regional Referral Hospital from July to October 2020.

\begin{tabular}{|c|c|c|c|c|c|c|}
\hline \multirow{3}{*}{ Variable } & \multirow{3}{*}{ Category } & \multicolumn{2}{|c|}{ Non-adherence } & \multicolumn{3}{|c|}{ Multivariate analysis } \\
\hline & & No & Yes & AOR & $95 \% \mathrm{Cl}$ & $P$ \\
\hline & & $\mathbf{N}(\%)$ & $\mathbf{N}(\%)$ & & & \\
\hline \multirow[t]{2}{*}{ Gender } & Male & $48(53.9)$ & $\begin{array}{l}41 \\
(46.1)\end{array}$ & 1 & & \\
\hline & Female & $111(66.1)$ & $\begin{array}{l}57 \\
(33.9)\end{array}$ & 0.57 & $\begin{array}{l}0.312- \\
1.044\end{array}$ & 0.069 \\
\hline \multirow[t]{3}{*}{ Age } & $18-24$ & $7(77.8)$ & $\begin{array}{l}2 \\
(22.2)\end{array}$ & 1 & & \\
\hline & $25-60$ & $\begin{array}{l}120 \\
(72.3)\end{array}$ & $\begin{array}{l}46 \\
(27.7)\end{array}$ & 1.19 & $\begin{array}{l}0.204- \\
6.962\end{array}$ & 0.845 \\
\hline & Above 60 & $32(39.0)$ & $\begin{array}{l}50 \\
(61.0)\end{array}$ & 6.29 & $\begin{array}{l}1.009- \\
39.241\end{array}$ & 0.049 \\
\hline \multirow[t]{4}{*}{ Education } & Primary & $71(67.0)$ & $\begin{array}{l}35 \\
(33.0)\end{array}$ & 1 & & \\
\hline & Not educated & $32(53.3)$ & $\begin{array}{l}28 \\
(46.8)\end{array}$ & 1.36 & $\begin{array}{l}0.634- \\
2.933\end{array}$ & 0.426 \\
\hline & High school & $36(64.3)$ & $\begin{array}{l}20 \\
(35.7)\end{array}$ & 1.84 & $\begin{array}{l}0.828- \\
4.073\end{array}$ & 0.135 \\
\hline & Tertiary & $20(57.1)$ & $\begin{array}{l}15 \\
(42.9)\end{array}$ & 1.88 & $\begin{array}{l}0.663- \\
5.317\end{array}$ & 0.235 \\
\hline \multirow[t]{2}{*}{$\begin{array}{l}\text { Duration of Diabetes } \\
\text { (years) }\end{array}$} & 5 years \& below & $\begin{array}{l}110 \\
(68.8)\end{array}$ & $\begin{array}{l}50 \\
(31.2)\end{array}$ & 1 & & \\
\hline & Above 5 years & $49(50.5)$ & $\begin{array}{l}48 \\
(49.5)\end{array}$ & 1.87 & $\begin{array}{l}1.034- \\
3.392\end{array}$ & 0.038 \\
\hline \multirow[t]{5}{*}{ Employment status } & Employed & $33(56.9)$ & $\begin{array}{l}25 \\
(43.1)\end{array}$ & 1 & & \\
\hline & farmers & $45(52.9)$ & $\begin{array}{l}40 \\
(47.1)\end{array}$ & 0.85 & $\begin{array}{l}0.344- \\
2.110\end{array}$ & 0.730 \\
\hline & Entrepreneur & $30(71.4)$ & $\begin{array}{l}12 \\
(28.6)\end{array}$ & 0.57 & $\begin{array}{l}0.212- \\
1.519\end{array}$ & 0.259 \\
\hline & Un employed & $45(71.4)$ & $\begin{array}{l}18 \\
(28.6)\end{array}$ & 0.37 & $\begin{array}{l}0.139- \\
0.977\end{array}$ & 0.045 \\
\hline & Others & $6(66.6)$ & $\begin{array}{l}3 \\
(33.3)\end{array}$ & 0.21 & $\begin{array}{l}0.036- \\
1.257\end{array}$ & 0.088 \\
\hline
\end{tabular}




\begin{tabular}{|c|c|c|c|c|c|c|}
\hline \multirow[b]{2}{*}{$\begin{array}{l}\text { Antidiabetic regimen } \\
\text { used }\end{array}$} & \multirow[b]{2}{*}{ Metformin } & \multicolumn{2}{|c|}{ Non-adherence } & \multicolumn{3}{|c|}{ Multivariate analysis } \\
\hline & & $24(66.7)$ & $\begin{array}{l}12 \\
(33.3)\end{array}$ & 1 & & \\
\hline & Insulin & $19(52.9)$ & $\begin{array}{l}17 \\
(47.2)\end{array}$ & 1.93 & $\begin{array}{l}0.652- \\
5.728\end{array}$ & 0.234 \\
\hline & Metformin \& insulin & $31(72.1)$ & $\begin{array}{l}12 \\
(27.9)\end{array}$ & 0.73 & $\begin{array}{l}0.243- \\
2.178\end{array}$ & 0.570 \\
\hline & $\begin{array}{l}\text { Metformin \& } \\
\text { Glibenclamide }\end{array}$ & $85(59.9)$ & $\begin{array}{l}57 \\
(40.1)\end{array}$ & 1.42 & $\begin{array}{l}0.582- \\
3.466\end{array}$ & 0.440 \\
\hline
\end{tabular}

\section{Surrogate makers}

Mean $\mathrm{HbA1c}$ of those who were non-adherent was $9.43 \% \pm 2.70$; which was not significantly higher ( $\mathrm{p}$ value $=0.266$ ) compared to $\mathrm{HbA} 1 \mathrm{c}$ of $9.07 \pm 2.39$ of those who were adherent. However, their mean FBG was significantly higher than that of those who were adherent $(10.01 \pm 5.26$ vs $8.33 \pm 5.04$; $p$ value $=$ 0.011) (Table 4).

Table 6

Results of student t-test for Surrogate Markers in relation to antidiabetic medication nonadherence among study participants in the Diabetes clinic at Mbarara Regional Referral Hospital between July to October 2020 .

\begin{tabular}{|c|c|c|c|c|c|c|c|}
\hline \multirow{3}{*}{ Variable } & \multicolumn{7}{|c|}{ Non-adherence } \\
\hline & \multicolumn{3}{|l|}{ YES } & \multicolumn{3}{|l|}{ NO } & \multirow[t]{2}{*}{ P-Value } \\
\hline & Mean & SD & $\mathrm{Cl}$ & Mean & SD & $\mathrm{Cl}$ & \\
\hline HbA1C (\%) & 9.43 & 2.70 & $8.89-9.98$ & 9.07 & 2.39 & $8.70-9.45$ & 0.266 \\
\hline FBG (Mmol/l ) & 10.01 & 5.26 & $8.95-11.06$ & 8.33 & 5.04 & $7.54-9.12$ & 0.011 \\
\hline
\end{tabular}

\section{Discussion}

A total of 257 study participants were recruited with $100 \%$ response rate. Majority $(253,98.4 \%)$ of participants had T2DM and almost two thirds $(160,62.3 \%)$ had been diagnosed with diabetes mellitus for less than five years. Majority $(214,83.3 \%)$ of the participants had comorbidities of which hypertension $(143,66.8 \%)$ was the most common. One quarter $(50,25.5 \%)$ of the participants reported that drug(s) being expensive was the cause of antidiabetic medication non-adherence and less than one quarter (38, $19.4 \%$ ) ascribed non-adherence to not understanding instruction(s) on how to take prescribed medicine(s). More than one third $(98,38.1 \%)$ of the participants were non-adherent to their antidiabetic medication. Multivariate logistic regression revealed that age above 60 years $(A O R=6.26,95 \% \mathrm{Cl}=1.009-$ 
$39.241, \mathrm{P}=0.049)$ and duration of diabetes mellitus of above 5 years since diagnosis $(A O R=1.87,95 \% \mathrm{Cl}$ $=1.034-3.391, P=0.038)$ were independently associated with antidiabetic medication non adherence.

More than one-third (38.1\%) of participants in our study were determined to be non-adherent to their antidiabetic medication. Reasons for non-adherence ranged from; drugs being expensive for patients to buy on daily basis (25.5\%), lack of understanding of prescription instructions (19.4\%), unavailability of free antidiabetic medicines in public hospitals $(16.3 \%)$, forgetfulness $(12.2 \%)$ and patients' preference for not following prescription instructions (12.2\%). The findings in our study, revealed a higher prevalence of non-adherence to antidiabetic medication compared to similar studies in UAE, Egypt, Ghana, Mulago Hospital in Uganda, and Tanzania; which reported between $16.0 \%$ and $31.5 \%$ prevalence of medication non-adherence among diabetes mellitus $(4,5,13,17,18)$. Perhaps this may be due to differences in the study populations and the study settings of different studies. Findings of our study in which majority $(214,83.3 \%)$ of the participants had comorbidities are comparable to the results of the study by (19)where comorbidities were significantly linked to antidiabetic medication non-adherence. The explanation could be that, the number of drugs taken by each patient is dependent on comorbidities. Therefore, a patient with a complex regimen is challenged to continue adhering to all prescribed medications. Multiple medications could contribute to non-adherence since such patients have divided commitment to managing several comorbidities. Also, since majority of our study population had low or no formal education (166,64.7\%), this could explain the high levels of non-adherence to antidiabetic medications as further reported by previous studies in Ghana (20). The reason for this finding could be that as the antidiabetic regimen becomes complex, those with higher level of education are expected to easily understand such and thus effectively follow their treatment regimen. However, the findings of our study are notably lower, with the prevalence of non-adherence to antidiabetic medication at $38.1 \%$ compared to 48-68.8\% prevalence, as reported from multiple studies in Bosnia \& Herzegovina, India, Saudi Arabia and Ethiopia $(8,9,21,22)$. This difference might be that in our study, patients regularly received free antidiabetic medications whenever available at the Diabetes clinic. Free supply of antidiabetic medicines is believed to reduce the level of non-adherence among patients (Kalyango et al., 2008). The other reason might be that in our study, patients were well organized in a patient association that provided psychological support and counseling to patients. The lower prevalence of non-adherence in our study settings could also be explained by the lower levels of forgetfulness (12.3\%) and lack of finances $(25.5 \%)$, as compared to similar studies in Sudan, Ghana, and Saudi Arabia, which reported $30.7-66.7 \%$, and $23.8-25.6 \%$ frequencies of forgetfulness and lack of finances, respectively $(17,23,24)$. Since our study population majorly consisted of the female gender $(168,65.37 \%)$; findings from our study revealed that the female gender was $57 \%$ less likely to non-adhere to their antidiabetic medication (AOR $=0.5795 \% \mathrm{Cl}=0.312-1.044 \mathrm{p}$-value $=0.069)$. This is in agreement with findings by $(25)$, stating that the female gender were more adherent to their antidiabetic medications, possibly due to the reason that most females spent most of their time at home and are thus more likely to follow prescription instructions agreed upon with the prescribers due to convenient home environment.

Multivariate logistic regression revealed that, increasing age above 24 years had a positive association with likelihood of non- adherence to antidiabetic medication. Age groups between 24-60 years and 
above 60 years were 1.1 times $(\mathrm{AOR}=1.19,95 \% \mathrm{Cl}=0.204-6.962, \mathrm{p}$-value $=0.845)$ and 6.2 times $(\mathrm{AOR}=$ $6.2995 \% \mathrm{Cl}=1.009-39.241 \mathrm{p}$-value $=0.049)$ more likely to be non-adherent to their antidiabetic medication, respectively. This finding in our study may be due to the fact that older adults are more likely to forget to take their medication and also older adults in our setting are less financially capable to access medications from drug outlets, as prescribed by the healthcare provider. This will as well explain the contrasting findings in a previous study in Ethiopia, where older adults were more likely to be adherent to their antidiabetic medication due to the possible difference in social-economic support to the elderly people (22). Duration of diabetes mellitus for more than 5years since diagnosis was also significantly associated with non-adherence to antidiabetic medication ( $A O R=1.87,95 \% \mathrm{Cl}=1.034-3.391, \mathrm{P}=0.038$ ). this is in agreement with the study from Saudi Arabia $(3,24)$. It demonstrates that, the longer the duration of diabetes mellitus, the more the rate of non-adherence. This finding may be explained by the anxiety and fear that patients experience during early years following diagnosis and thus become committed to managing their disease but the commitment gradually wears out as they adapt to the burden of the disease and non-adherence emerges. Surprisingly, in the current study, being unemployed (AOR $=0.37$, $95 \% \mathrm{Cl}=0.139-0.977, \mathrm{p}$-value $=0.045$ ) was protective and therefore less likely to lead to non-adherence to antidiabetic medication. The explanation could be that unemployed patients usually have enough free time and were thus more likely to remember to take their antidiabetic medication.

Mean $\mathrm{HbA} 1 \mathrm{c}$ of those who were non adherent was $9.43 \%$, their mean FBG was $10.01 \mathrm{Mmol} / \mathrm{l}, \mathrm{P}=0.01$ and was significantly associated with antidiabetic medication non-adherence

\section{Limitations}

Due to Covid 19 pandemic, some patients were not comfortable with participating in research; this could have affected the quality of interaction and interview.

There was a challenge of space within the Diabetes mellitus clinic premise, with limited privacy during interviews. This could have compromised the privacy standards and thus impacted on the quality of patient responses.

Some study participants may have failed to recall accurately (recall bias) their previous experiences regarding adherence to their antidiabetic medication, thus ended up giving incomplete information that may have compromised study outcomes.

\section{Strength of the study}

Response rate was $100 \%$ which most likely gave a better estimate of the study outcome variables.

\section{Conclusion}

More than one third of the study participants were non-adherent to their antidiabetic medication, with medication expense being the most frequent reason mentioned for non-adherence to antidiabetic 
medication. Patients aged 60 years and above, and those having more than 5 years of disease since diagnosis, were 6.2 and 1.1 times more likely to be non-adherent to their antidiabetic medications.

\section{Abbreviations}

CADS, Conventional Antidiabetic Drugs; CVS, Cardiovascular System; DGRT, Directorate of Postgraduate Training and Research; DKA, Diabetic Ketoacidosis; DM, Diabetes Mellitus; FBG Fasting Blood Glucose; HbA1c, Glycated Hemoglobin; HHS, Hyperosmolar Hyperglycemic Non-Ketotic Syndrome; MRRH, Mbarara Regional Referral Hospital; MUST, Mbarara University of Science and Technology; REC, Research Ethics Committee; RBG, Random Blood Glucose; T2DM, Type 2 Diabetes mellitus; TIDM, Type 1 Diabetes mellitus; AOR, Adjusted Odds Ratios; Cl, Confidence Interval; OR Odds Ratios; WHO, World Health Organization.

\section{Declarations}

\section{Ethics approval and consent to participate}

Voluntary recruitment was done and an informed consent was signed by study participants. Informed consent from study participants was obtained after full explanation of the details of the study to them in English and interpreted to local language (Runyankole) for those participants who did not understand English. Participants were not forced to enroll themselves if they did not want to. Study participants were also at liberty to withdraw from the study at any time as they wished without coercion or compromise of care they were entitled to.

\section{Consent for publication}

All authors agreed to submission of this manuscript for publication in addition to the consent to publish which was included in the informed consent form which attained ethical and participant approval.

\section{Availability of data and material}

The datasets used and analyzed during the current study are available from the corresponding author on reasonable request.

\section{Competing interests}

The authors declare that they have no conflicts of interest for this work.

\section{Author Contributions}

All authors made significant contributions to conception and design, acquisition of data, analysis and interpretation of data; took part in drafting the article and revising it critically for important scholarly 
content; agreed to submit to the current journal; gave final approval of the version to be published; and agreed to take responsibility for all aspects of the work.

\section{Acknowledgement}

We would like to thank all members of staff at the Department of Pharmacy, Diabetes clinic-MRRH, DGRT of Mbarara University of Science and Technology and all individuals who have supported us towards completion of this research project.

\section{Funding}

This research work was funded by personal resources

\section{References}

1. Al-Lawati JA. Diabetes mellitus: a local and global public health emergency! Oman medical journal. 2017;32(3):177.

2. Yazew KG, Walle TA, Azagew AW. Prevalence of anti-diabetic medication adherence and determinant factors in Ethiopia: a systemic review and meta-analysis, 2019. International Journal of Africa Nursing Sciences. 2019;11:100167.

3. Bagonza J, Rutebemberwa E, Bazeyo W. Adherence to anti diabetic medication among patients with diabetes in eastern Uganda; a cross sectional study. BMC health services research. 2015;15(1):168.

4. Fedrick F, Temu M. Factors contributing to non-adherence to diabetes treatment among diabetic patients attending clinic in Mwanza city. East African journal of public health. 2012;9(3):90-5.

5. Heissam K, Abuamer Z, El-Dahshan N. Patterns and obstacles to oral antidiabetic medications adherence among type 2 diabetics in Ismailia, Egypt: a cross section study. The Pan African Medical Journal. 2015;20.

6. Guerci B, Chanan N, Kaur S, Jasso-Mosqueda JG, Lew E. Lack of treatment persistence and treatment nonadherence as barriers to glycaemic control in patients with type 2 diabetes. Diabetes Therapy. 2019;10(2):437-49.

7. Venkatesan M, Dongre AR, Ganapathy K. A community-based study on diabetes medication nonadherence and its risk factors in rural Tamil Nadu. Indian journal of community medicine: official publication of Indian Association of Preventive \& Social Medicine. 2018;43(2):72.

8. Bhattarai B, Bista B, Shrestha S, Budhathoki B, Dhamala B. Contributing Factors of Non-Adherence to Treatment among the Patients with Type II Diabetes Mellitus. Journal of Manmohan Memorial Institute of Health Sciences. 2019;5(1):68-78.

9. Khan AR, Lateef ZNA-A, Al Aithan MA, Bu-Khamseen MA, Al Ibrahim I, Khan SA. Factors contributing to non-compliance among diabetics attending primary health centers in the Al Hasa district of Saudi Arabia. Journal of Family and Community Medicine. 2012;19(1):26. 
10. Afriyie G. Non-Adherence to Medication and Associated Factors among Type 2 Diabetes Mellitus Patients Attending Tema General Hospital: University of Ghana; 2019.

11. Kusa W, Tolessa D, Abdeta T. Type II DM Medication Non-Adherence in Adama Hospital Medical College, Central Ethiopia. East African Journal of Health and Biomedical Sciences. 2019;3(1):31-8.

12. Kassahun A, Fanta Gashe EM, Rike WA. Nonadherence and factors affecting adherence of diabetic patients to anti-diabetic medication in Assela General Hospital, Oromia Region, Ethiopia. Journal of pharmacy \& bioallied sciences. 2016;8(2):124.

13. Kalyango JN, Owino E, Nambuya AP. Non-adherence to diabetes treatment at Mulago Hospital in Uganda: prevalence and associated factors. African health sciences. 2008;8(2).

14. Naing L, Winn T, Rusli B. Practical issues in calculating the sample size for prevalence studies. Archives of orofacial Sciences. 2006;1:9-14.

15. Chan A, Horne R, Hankins M, Chisari C. The Medication Adherence Report Scale: A measurement tool for eliciting patients' reports of nonadherence. Br J Clin Pharmacol 2020;86:1281-8.

16. World Medical Association. WMA DECLARATION OF HELSINKI - ETHICAL PRINCIPLES FOR MEDICAL RESEARCH INVOLVING HUMAN SUBJECTS 2018 [cited 2020 20th of December]. Available from: https://www.wma.net/policies-post/wma-declaration-of-helsinki-ethical-principles-for-medicalresearch-involving-human-subjects/.

17. Arifulla M, Lisha Jenny J, Sreedharan J, Muttappallymyalil J, Basha SA. Patients' adherence to antidiabetic medications in a hospital at Ajman, UAE. The Malaysian journal of medical sciences: MJMS. 2014;21(1):44.

18. Amaltinga APM. Non adherence to diabetic medication among diabetic patients, a case study of Dormaa Hospital Ghana. Science Journal of Public Health. 2017;5(2):88-97.

19. Alqarni AM, Alrahbeni T, Al Qarni A, Al Qarni HM. Adherence to diabetes medication among diabetic patients in the Bisha governorate of Saudi Arabia-a cross-sectional survey. Patient preference and adherence. 2019;13:63.

20. Afaya RA, Bam V, Azongo TB, Afaya A, Kusi-Amponsah A, Ajusiyine JM, et al. Medication adherence and self-care behaviours among patients with type 2 diabetes mellitus in Ghana. PloS one. 2020;15(8):e0237710.

21. Horvat O, Popržen J, Tomas A, Kusturica MP, Tomić Z, Sabo A. Factors associated with nonadherence among type 2 diabetic patients in primary care setting in eastern Bosnia and Herzegovina. Primary care diabetes. 2018;12(2):147-54.

22. Abate TWg. Medication non-adherence and associated factors among diabetes patients in Felege Hiwot Referral Hospital, Bahir Dar city administration, Northwest Ethiopia. BMC research notes. 2019;12:1-6.

23. El-Hadiyah TM, Madani AM, Abdelrahim HM, Yousif AK. Factors affecting medication non adherence in type 2 Sudanese diabetic patients. Pharmacology \& Pharmacy. 2016;7(4):141-6.

24. Almaghaslah D, Abdelrhman AK, AL-Masdaf SK, Majrashi LM, Matary BM, Asiri WM, et al. Factors contributing to non-adherence to insulin therapy among type 1 and type2 diabetes mellitus patients 
in Asser region, Saudi Arabia. 2018.

25. Gelaw BK, Mohammed A, Tegegne GT, Defersha AD, Fromsa M, Tadesse E, et al. Nonadherence and contributing factors among ambulatory patients with antidiabetic medications in Adama Referral Hospital. Journal of diabetes research. 2014;2014.

\section{Figures}

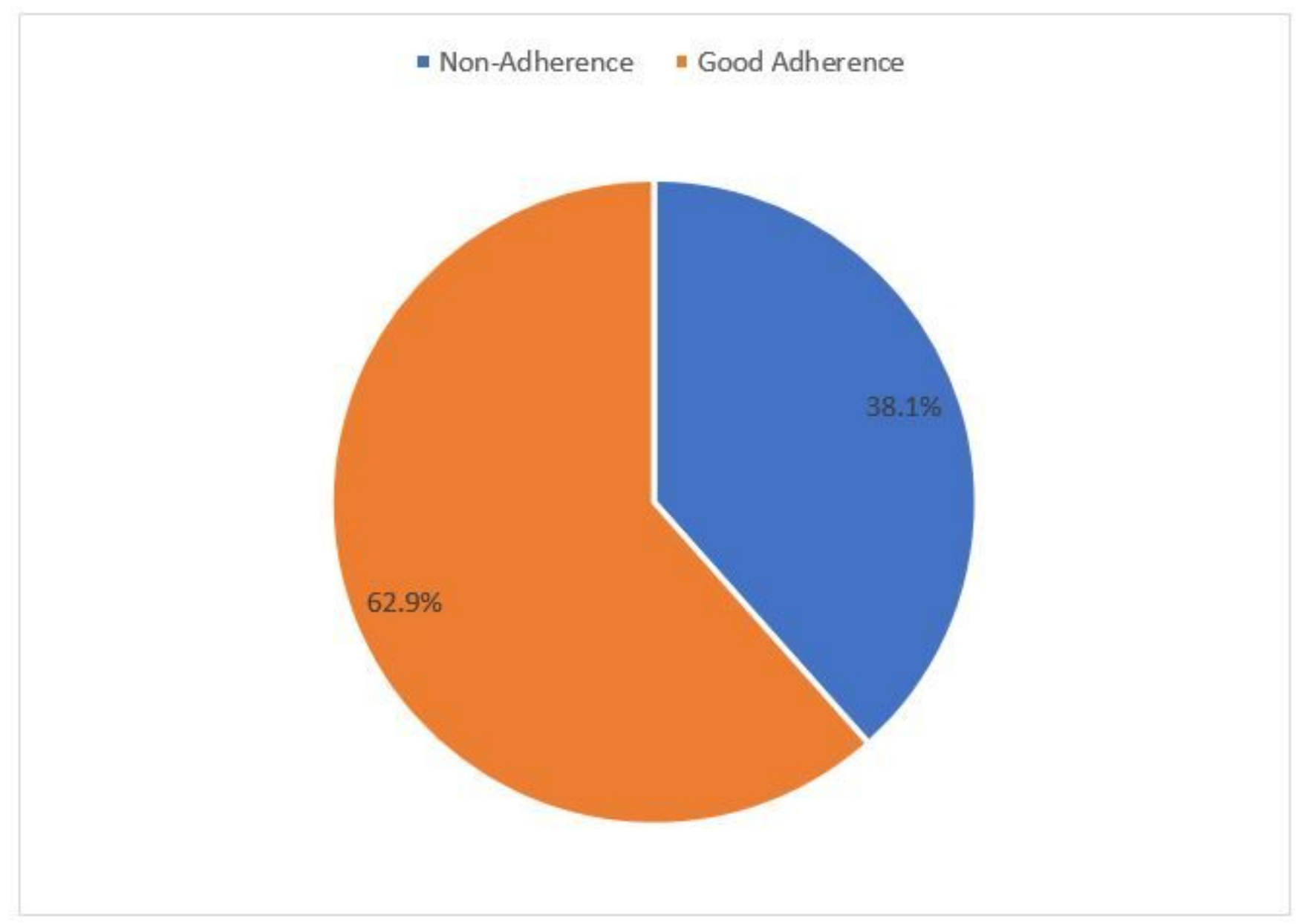

\section{Figure 1}

Pie chart showing the level of antidiabetic medication non-adherence among participants in the Diabetes mellitus clinic at Mbarara Regional Referral Hospital from July to October 2020 